\title{
FluXOR: Detecting and Monitoring Fast-flux Service Networks
}

\section{Emanuele Passerini, Roberto Paleari, Lorenzo Martignoni, Danilo Bruschi}

\author{
DIMVA 2008
}




\section{Botnets}

\section{What is a botnet?}

- a network of infected machines (bots) used simultaneously to achieve the same purpose

- different purposes: spam, DDoS, phishing, scam, massive SQL injection, ...
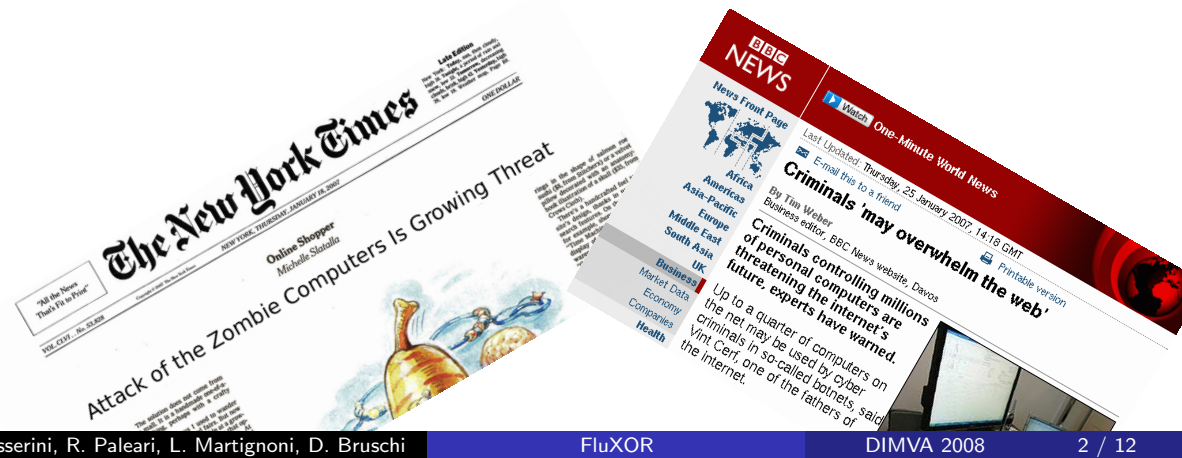


\section{Botnets}

\section{What is a botnet?}

- a network of infected machines (bots) used simultaneously to achieve the same purpose

- different purposes: spam, DDoS, phishing, scam, massive SQL injection, ...

\section{Fast-flux service networks}

- a new ( 2007) technique to maximize botnets availability

- simple idea: add an additional indirection layer (i.e., proxy) between victims and controlling elements 


\section{Fast-flux botnets}

Architecture

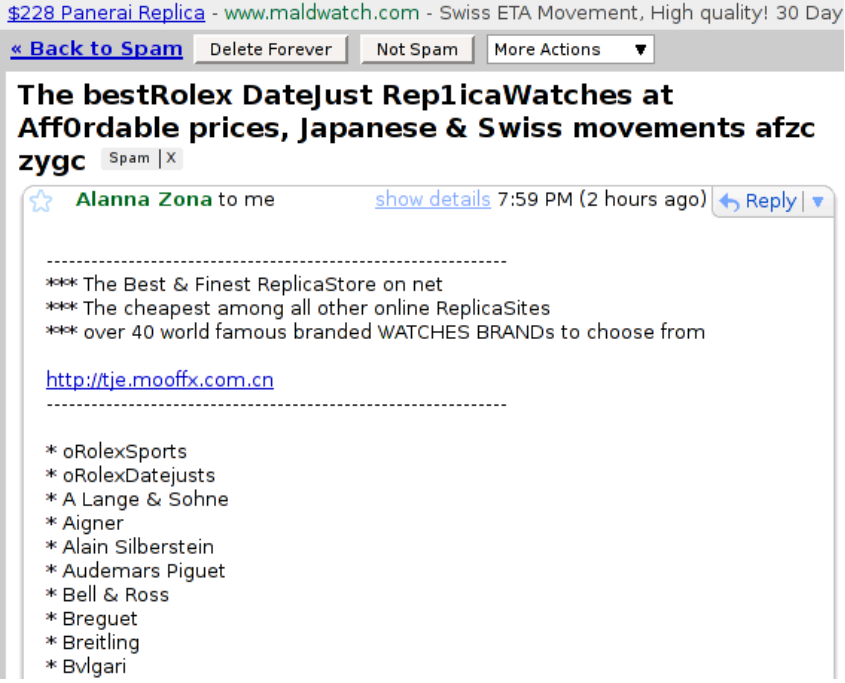

* Breguet

* Breitling

* Bvlgari 


\section{Fast-flux botnets}

\section{Architecture}
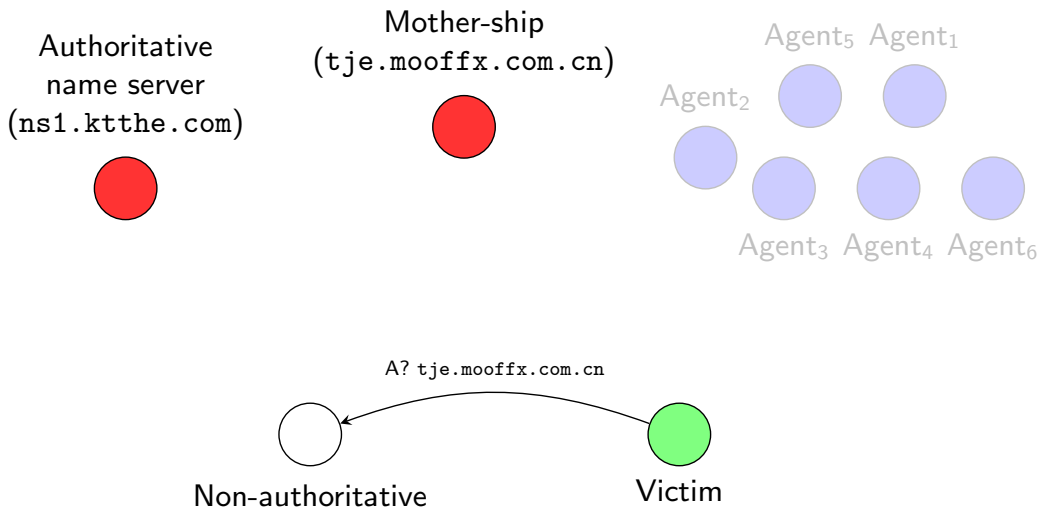

Non-authoritative name server 


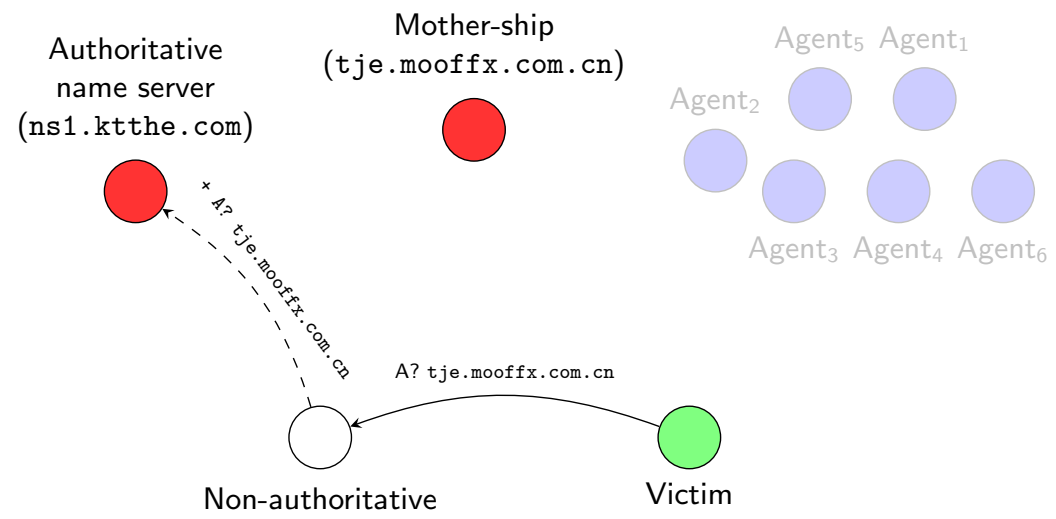

name server 


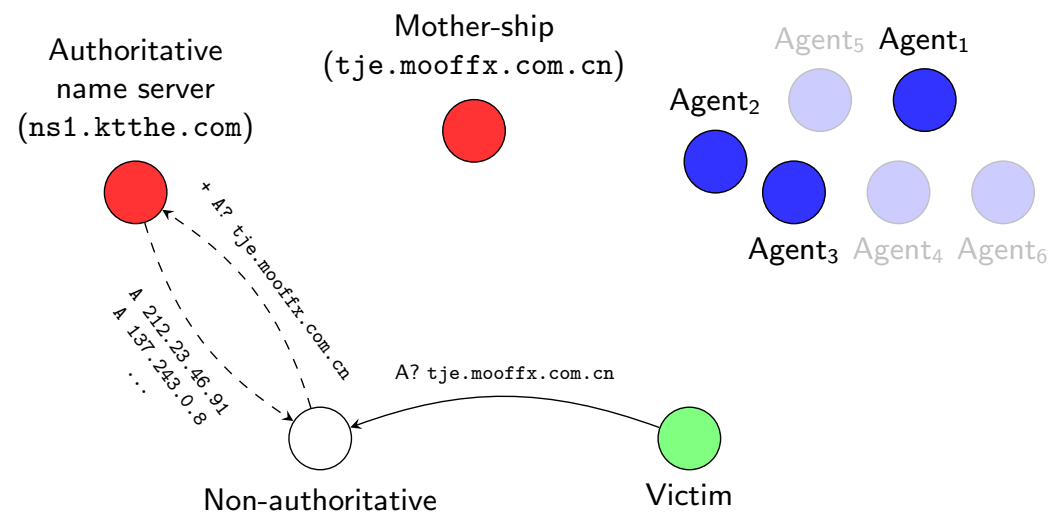

name server 


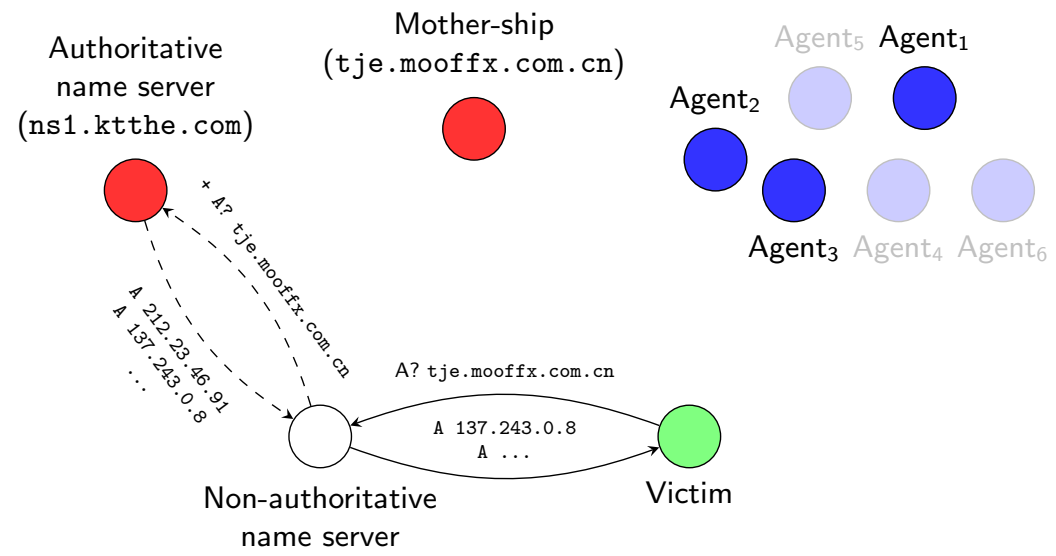




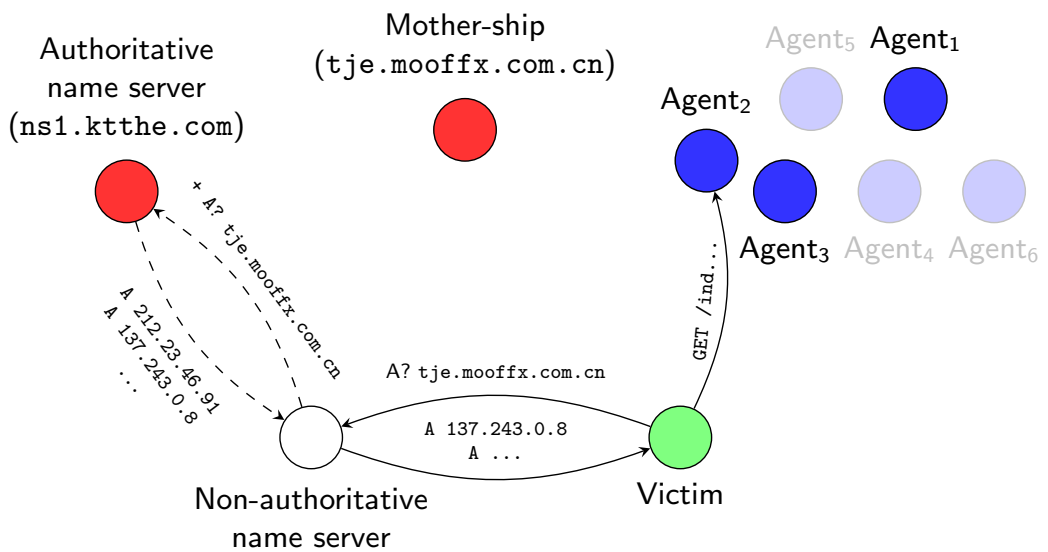




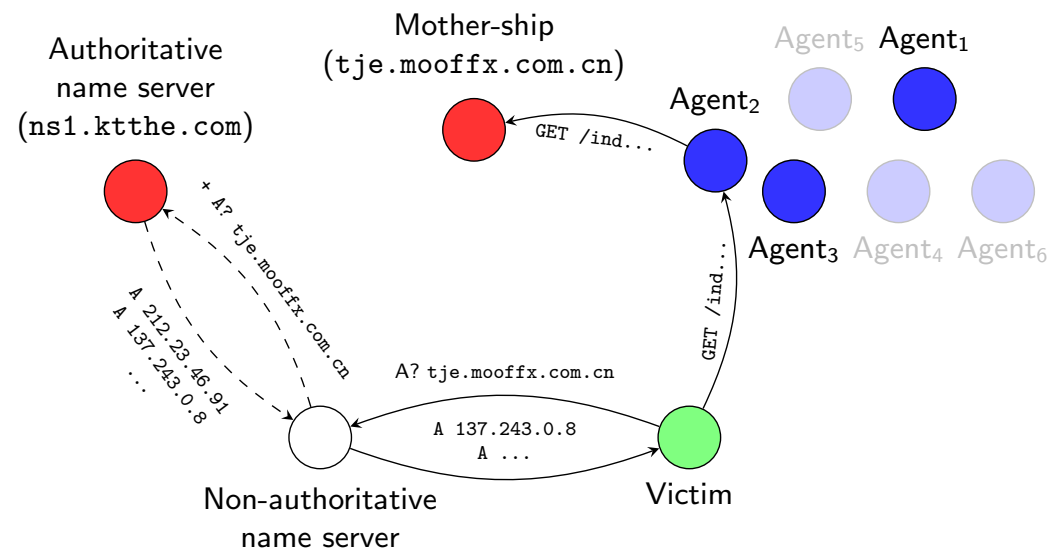




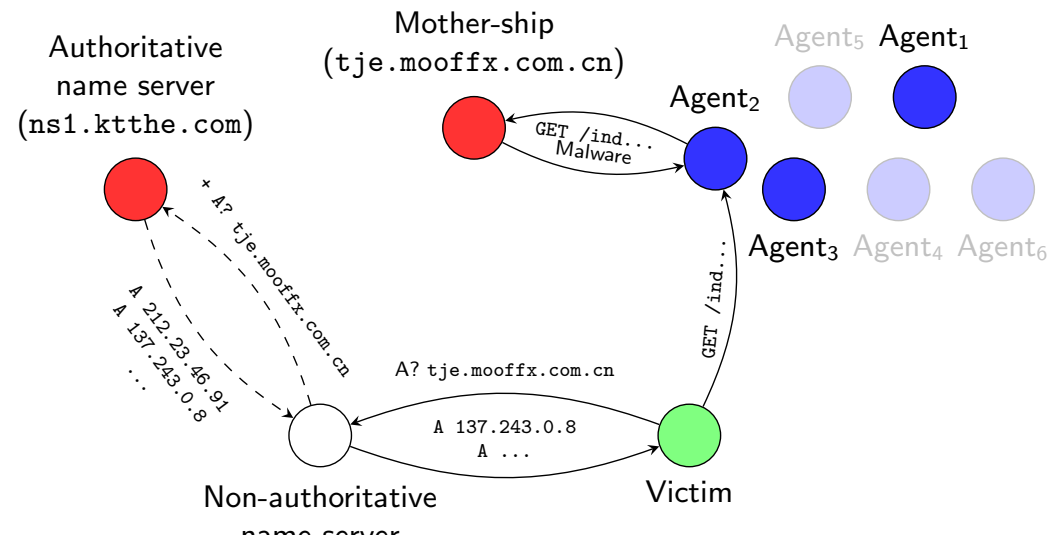

name server 


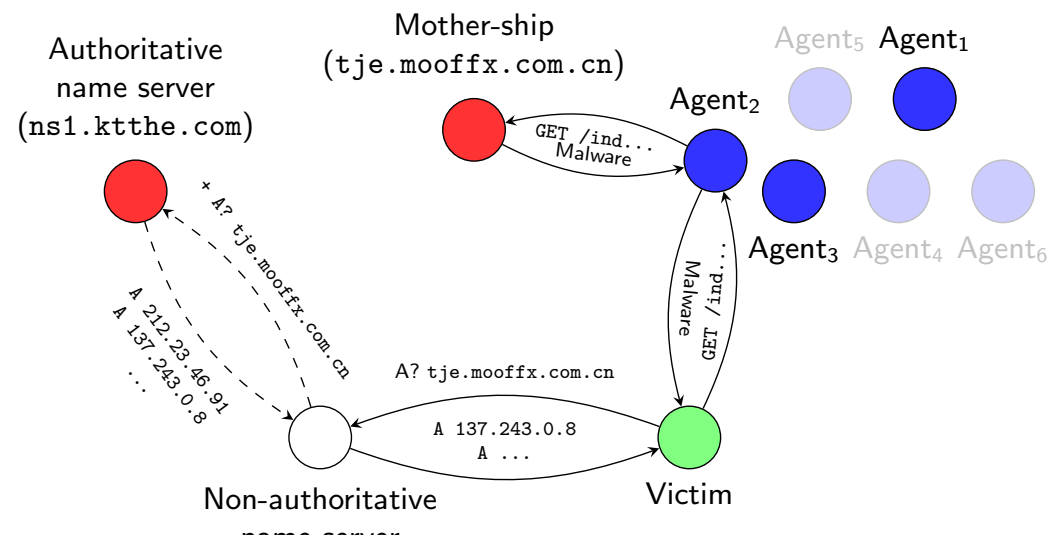

name server 


\section{Fast-flux botnets}

\section{Characteristics}

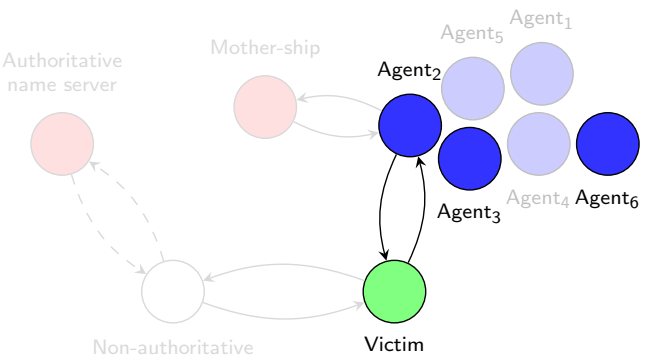

- off-line, disinfected, and faulty bots (or agents) are immediately replaced by others

- Warezov/Storm networks have millions of agents!

- Storm: 1 billion spam messages during a six-weeks attack 


\section{Fast-flux botnets}

\section{Characteristics}

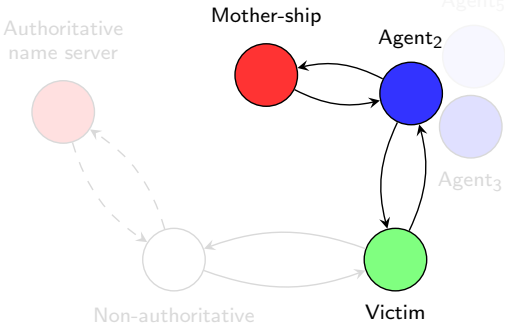

identity of the core components of the architecture (e.g., mothership) is hidden to the victims 


\section{Fast-flux botnets}

\section{Characteristics}

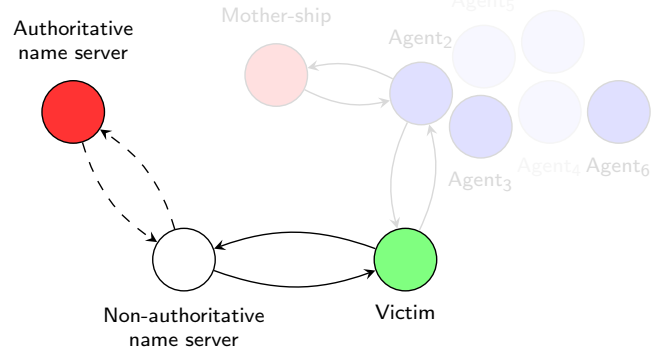

- multiple FQDNs can be associated with the same fast-flux service network

- it is not enough to close malicious FQDN! 


\section{Fast-flux botnets}

\section{Characteristics}

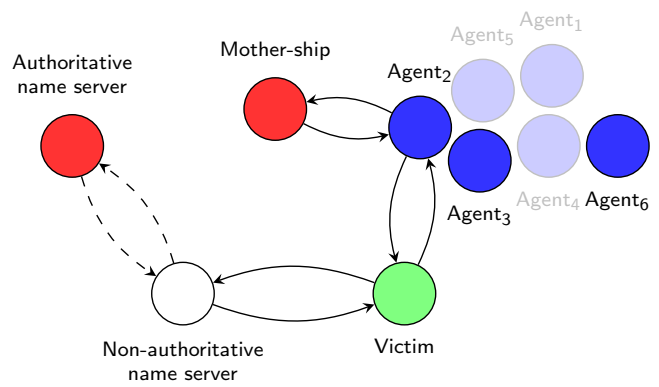

Real impact

- The average lifetime of the scam site becomes months instead of days!

- The only way shut down scam site is to clean all agents 


\section{Our contribution}

\section{Observation}

- a fast-flux service network has multiple distinguishing features

- taken singularly are not enough to distinguish between benign and malicious hostnames

\section{Idea: FluXOR}

- monitor the suspicious hostname for a small period of time to collect distinguishing features, behaving like a recidivious victim

- combine features to distinguish between benign and malicious domains

- monitor malicious domains to enumerate all infected agents 


\section{Features of fast-flux service networks}

\section{Domain}

- Domain age

- Domain registrar

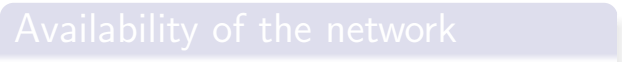

Heterogeneity of the agents

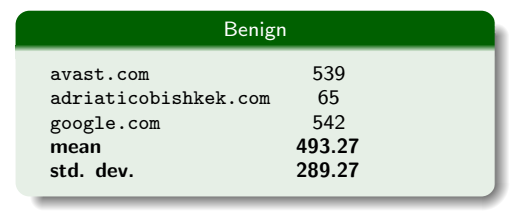

\begin{tabular}{|lc|}
\hline \multicolumn{2}{|c|}{ Malicious } \\
\hline eveningher.com & 18 \\
factvillage.com & 2 \\
doacasino.com & 2 \\
mean & 4.85 \\
std. dev. & 4.9 \\
\hline
\end{tabular}




\section{Features of fast-flux service networks}

\section{Domain}

- Domain age

- Domain registrar

Availability of the network

Heterogeneity of the agents

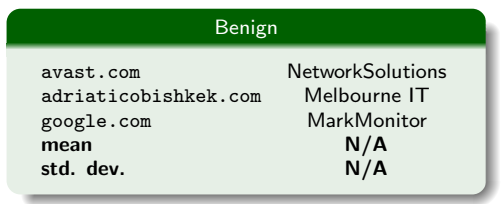

\section{Malicious}

eveningher.com

factvillage.com doacasino.com

mean

std. dev.
PayCenter

PayCenter NameCheap

N/A

N/A 


\section{Features of fast-flux service networks}

\section{Domain}

- Domain age

- Domain registrar

Availability of the network

- \# of DNS records of type "A"

- TTL of DNS resource records

Heterogeneity of the agents

\begin{tabular}{|lc|}
\hline \multicolumn{2}{|c|}{ Benign } \\
\hline avast.com & 12 \\
adriaticobishkek.com & 21 \\
google.com & 3 \\
mean & 2.86 \\
std. dev. & 3.89 \\
\hline
\end{tabular}

\section{Malicious}

$\begin{array}{lc}\text { eveningher.com } & 127 \\ \text { factvillage.com } & 117 \\ \text { doacasino.com } & 33 \\ \text { mean } & 98.13 \\ \text { std. dev. } & 37.27\end{array}$




\section{Features of fast-flux service networks}

\section{Domain}

- Domain age

- Domain registrar

Availability of the network

- \# of DNS records of type "A"

- TTL of DNS resource records

\begin{tabular}{|lc|}
\hline \multicolumn{2}{|c|}{ Benign } \\
\hline avast.com & 3600 \\
adriaticobishkek.com & 1200 \\
google.com & 300 \\
mean & $\mathbf{4 5 9 2 . 5 3}$ \\
std. dev. & $\mathbf{7 6 6 8 . 7 4}$ \\
\hline
\end{tabular}

\section{Malicious}

$\begin{array}{lc}\text { eveningher.com } & 300 \\ \text { factvillage.com } & 300 \\ \text { doacasino.com } & 180 \\ \text { mean } & \mathbf{2 6 1 . 4 9} \\ \text { std. dev. } & \mathbf{5 9 . 6 4}\end{array}$




\section{Features of fast-flux service networks}

\section{Domain}

- Domain age

- Domain registrar

Availability of the network

- \# of DNS records of type "A"

- TTL of DNS resource records

Heterogeneity of the agents

- \# of networks

. $\#$ of autonomous systems

- \# of resolved QDNs

- \# of assigned network names

- \# of organisations

\section{Benign}

avast.com

adriaticobishkek.com

google.com

mean

std. dev.

1

2

1.27

0.65

\section{Malicious}

$\begin{array}{lc}\text { eveningher.com } & 83 \\ \text { factvillage.com } & 81 \\ \text { doacasino.com } & 19 \\ \text { mean } & 63.75 \\ \text { std. dev. } & 23.91\end{array}$




\section{Features of fast-flux service networks}

\section{Domain}

- Domain age

- Domain registrar

Availability of the network

- \# of DNS records of type "A"

- TTL of DNS resource records

Heterogeneity of the agents

- \# of networks

- \# of autonomous systems

- * of resolved QDNs

- \# of assigned network names

* * of organisations

\section{Benign}

avast.com

3

adriaticobishkek. com

google.com

mean

std. dev.

1.11

0.36

\section{Malicious}

$\begin{array}{lc}\text { eveningher.com } & 49 \\ \text { factvillage.com } & 46 \\ \text { doacasino.com } & 14 \\ \text { mean } & \mathbf{3 8 . 3 6} \\ \text { std. dev. } & \mathbf{1 2 . 3 4}\end{array}$




\section{Features of fast-flux service networks}

\section{Domain}

- Domain age

- Domain registrar

Availability of the network

- \# of DNS records of type "A"

- TTL of DNS resource records

Heterogeneity of the agents

- \# of networks

- \# of autonomous systems

- \# of resolved QDNs

- \# of assigned network names

- \# of organisations 


\section{Overall architecture}

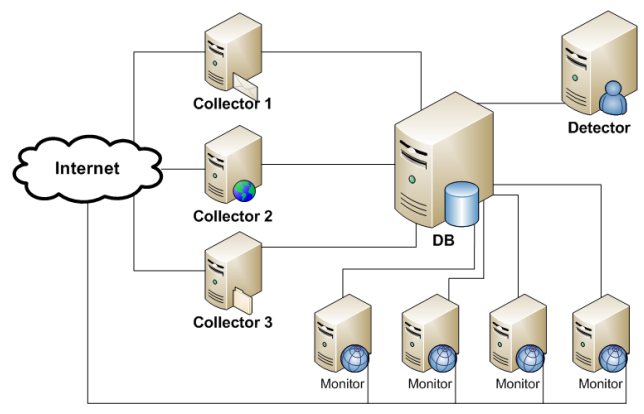

\section{Collector}

- harvests domain names from various sources (e.g., spam emails, DNS queries, ....)

- each collected domain name is flagged as suspicious 


\section{Overall architecture}

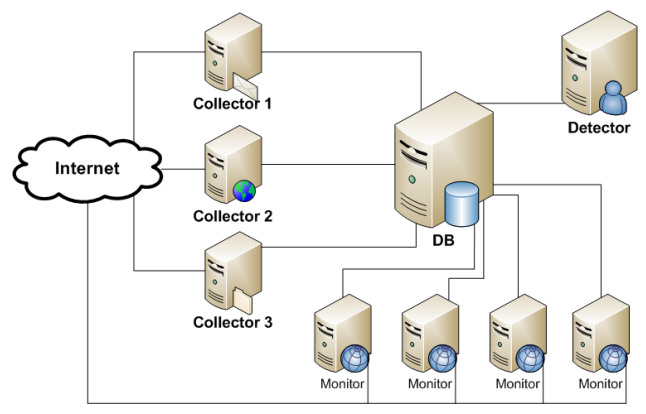

\section{Monitor}

- for each suspicious domain name, it collects characterizing features

- for each malicious domain name, it enumerates the IP addresses of the agents serving the network 


\section{Overall architecture}

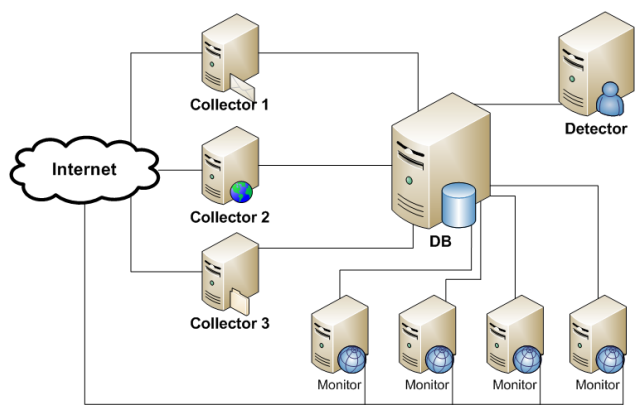

\section{Detector}

- automatic classification of domain names as malicious or benign

- combine collected features using naïve Bayesian classifier

- training sets: 50 benign + 58 malicious domains (manually classified) - automatic cross-validation 


\section{Implementation and deployment of the system}

Implementation \& deployment

- $\sim 2150$ lines of Python code + web interface

- MySQL DB (3 tables, the biggest one has $\sim 75$ millions tuples)

- distributed on 5 hosts (1 DB +1 collector +2 monitor +1 detector) 


\section{Experimental results}

\section{Detection accuracy}

Testing strategy:

- manual analysis of a random subset of the active domains

- just 1 hour to tell if a FQDN is malicious or not

\begin{tabular}{|l|c|}
\hline spam e-mails & 989530 \\
\hline FQDNs & 100508 \\
\hline benign FQDNs & 56920 \\
\hline inactive FQDNs & 35902 \\
\hline malicious FQDNs & $\mathbf{2 7 2 6 4}$ \\
\hline agents & $\mathbf{4 7 9 5 4 6}$ \\
\hline
\end{tabular}

Table: Summary of the results obtained using FluXOR since January 2008. 


\section{Experimental results}

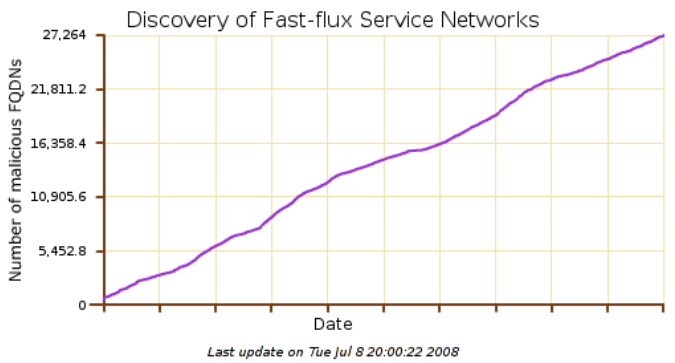




\section{Experimental results}
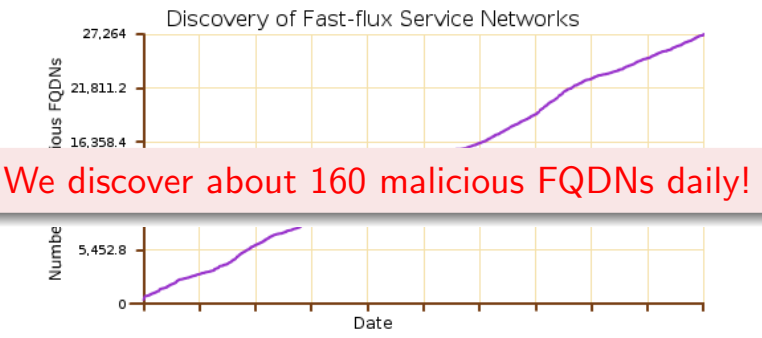

Last update on Tue Jul 8 20:00:22 2008 


\section{Experimental results}
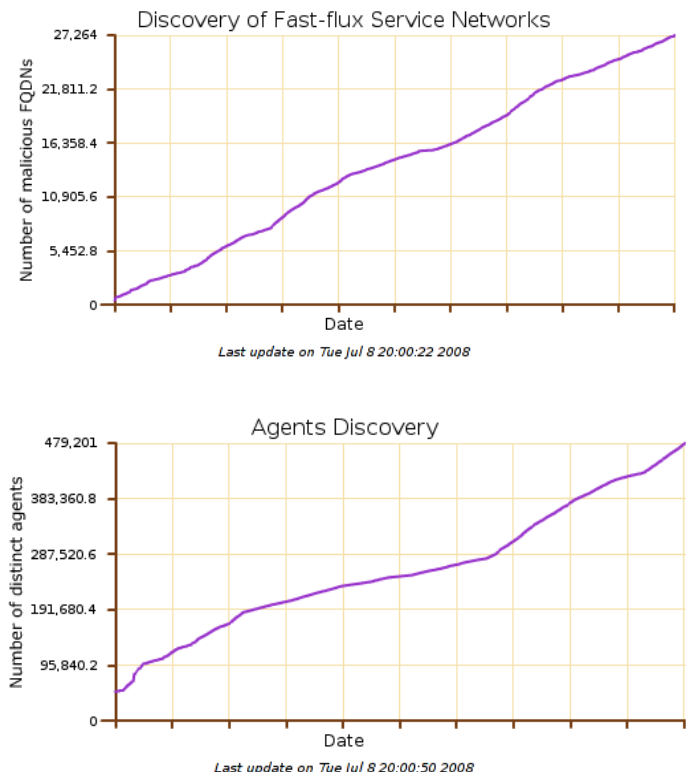


\section{Experimental results}
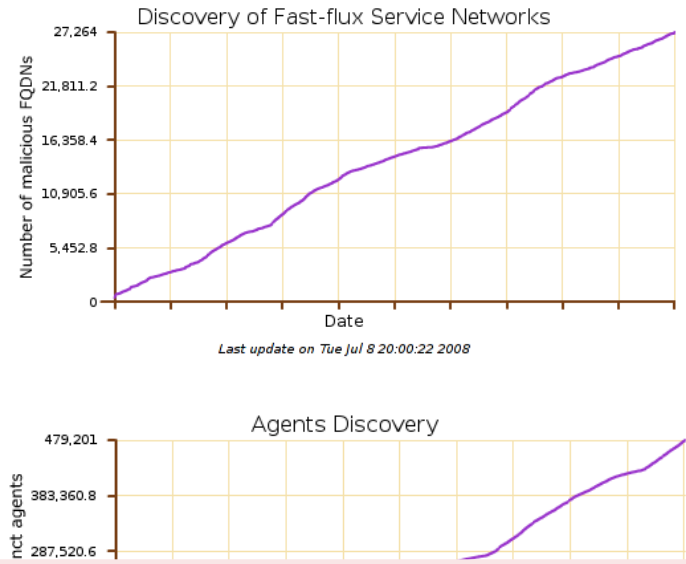

We discover more than 2200 new agents daily!

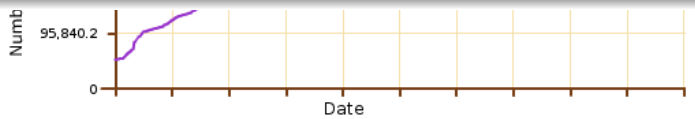

Last update on Tue Jul 8 20:00:50 2008 


\section{Conclusions}

\section{Contributions}

- identification of the features that characterize fast-flux botnets

- experimental system to monitor fast-flux service networks

- empirical analysis of the fast-flux phenomenon

\section{FluXOR: on-line web interface}

Real-time results are publicly available on-line at:

$$
\text { http://fluxor.laser.dico.unimi.it/ }
$$

Please wait until this afternoon: we have a (planned) blackout right now at our department in Milan ;-) 


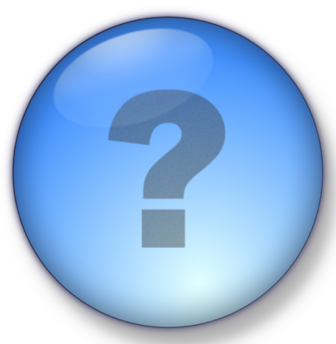

Questions?

$$
\text { http://fluxor.laser.dico.unimi.it/ }
$$

The average system load is 9.78 , we need a sponsor!! 